\title{
Adaptive Sliding Mode Control Using Robust Feedback Compensator for MEMS Gyroscope
}

\author{
Juntao Fei and Dan Wu \\ Jiangsu Key Laboratory of Power Transmission and Distribution Equipment Technology, \\ College of Computer and Information, Hohai University, Changzhou 213022, China
}

Correspondence should be addressed to Juntao Fei; jtfei@yahoo.com

Received 2 May 2013; Revised 27 August 2013; Accepted 11 September 2013

Academic Editor: Dane Quinn

Copyright (c) 2013 J. Fei and D. Wu. This is an open access article distributed under the Creative Commons Attribution License, which permits unrestricted use, distribution, and reproduction in any medium, provided the original work is properly cited.

An adaptive sliding mode control using robust feedback compensator is presented for a MEMS gyroscope in the presence of external disturbances and parameter uncertainties. An adaptive controller with a robust term is used to improve the robustness of the control system and compensate the system nonlinearities. The proposed robust adaptive control can estimate the angular velocity and all the system parameters including damping and stiffness coefficients in the Lyapunov framework. In addition, standard adaptive control scheme without robust algorithm is compared with the proposed robust adaptive scheme in the aspect of numerical simulation and algorithm derivation. Numerical simulations show that the robust adaptive control has better robustness in the presence of external disturbances than the standard adaptive control.

\section{Introduction}

In recent years, MEMS gyroscopes are commonly used microsensors for measuring angular velocity because of their compact size, low cost, and high sensitivity. Most MEMS gyroscopes utilize electrostatic force and capacitive detection. However, there are some damping effects and cross-stiffness in the presence of fabrication imperfections, and the performance of MEMS deteriorates due to the effects of timevarying parameters, external disturbances, and quadrature errors. Therefore, the measurement of angular velocity and minimization of the cross-coupling in the MEMS gyroscope should be investigated and studied. In recent years, the applications of adaptive control and robust control have achieved great developments. To control the MEMS gyroscopes, people proposed advanced control methods such as adaptive control, which can modify its characteristics with the varying parameters. Arancibia et al. [1] presented an adaptive control scheme for laser-beam steering by a twoaxis MEMS mirror. A robust adaptive control strategy using a fuzzy compensator for MEMS triaxial gyroscope is proposed in [2]. A robust adaptive control for MEMS triaxial gyroscope is developed in [3]. Jagannathan and Hameed [4] presented an adaptive force-balancing control scheme with actuator limits for a MEMS $z$-axis gyroscope. Zhu et al. [5] developed an alternative for the control of parallel-plate electros actuators. Leland [6] derived and summarized an adaptive controller to control all modes of a vibrational MEMS gyroscope. Park and Horowitz [7] presented adaptive add-on control algorithms for the conventional modes of operation of MEMS $z$-axis gyroscopes. Wang et al. [8] presented a micromachined vibratory gyroscope with two proof masses to adaptively measure a rotation rate. Liu et al. [9] introduced the design of a digital closed drive loop for a MEMS vibratory packaged gyroscope. Salah et al. [10] developed a new control strategy to sense the time-varying angular rate for MEMS $z$ axis gyroscopes. Some design and control methods have been proposed for MEMS gyroscope in [11-15]. Liu [16] proposed a robust adaptive control for an uncertain mechanical system.

In this paper, robust adaptive control scheme which has the advantage of combining the tracking capability of adaptive control with the robustness of robust control is proposed for the control of MEMS gyroscope. In addition, in the presence of external disturbance and model uncertainty, robust control is incorporated into the adaptive control to improve the dynamic characteristics and the robustness of the 
control system. The robust term can maintain some characteristics of the control system in the presence of parameter perturbations.

The robust adaptive control scheme with an additional robust term is firstly applied to the MEMS gyroscopes to improve the transient performance. Moreover, a standard adaptive control without feedback robust algorithm is proposed to estimate angular velocity and all unknown parameters for the purpose of comparison. The novelty in the paper is that a feedback algorithm is incorporated into the robust adaptive controller to improve the robustness of the control system and minimize the cross-coupling between two axes. The stability and convergence of the closed-loop control system can be guaranteed.

This paper focuses on the design and stability analysis of the robust adaptive control for MEMS gyroscopes. Meanwhile, in order to discuss the effect of the proposed control scheme, a comparative study between the standard adaptive control and the robust adaptive control is conducted, thus evaluating the performance index such as the convergence of angular velocity, tracking error, and the robustness of the control system. The advantages of the proposed control scheme are summarized compared to existing ones.

(1) A feedback algorithm is incorporated into the robust adaptive controller to deal with nonlinearities of a system. The robust adaptive controller can improve the robustness of the MEMS and estimate angular velocity and all unknown system parameters correctly. Meanwhile, cross-coupling between two axes is minimized in the presence of external disturbances. A novel robust adaptive control scheme is proposed and used to estimate angular velocity and all uncertain parameters with improved transient performance.

(2) Using Lyapunov analysis, the stability and convergence of the control system can be guaranteed. The tracking errors all converge to zero, and all the unknown parameters including angular velocity converge to their true values. Incorporating feedback algorithm into a robust adaptive controller, the control system tracks the desired reference trajectory much more quickly compared with other ones.

This paper is organized as follows. In Section 2, the dynamics of MEMS gyroscopes sensor is introduced. In Section 3, the design of robust adaptive control for MEMS gyroscopes is proposed. In Section 4, simulation results are provided, and comparative study between robust adaptive control and standard adaptive control is implemented. Conclusions are provided in Section 5.

\section{Dynamics of MEMS Gyroscope}

The dynamics of MEMS gyroscope are described in this section. Dynamics of a MEMS gyroscope are derived from Newton's law in the rotating frame.

In a $z$-axis gyroscope, by supposing the stiffness of spring in $z$ direction much larger than that in the $x, y$ directions, motion of poof mass is constrained to only along the $x$ $y$ plane as shown in Figure 1. Assuming that the measured

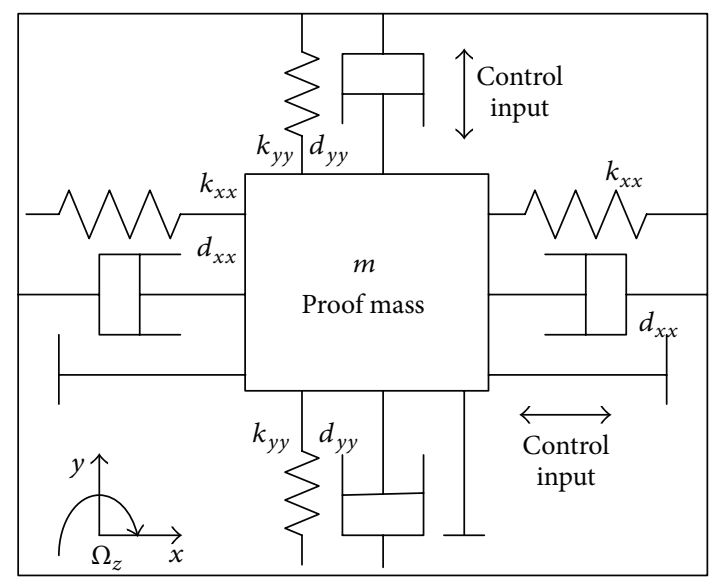

Figure 1: Simplified model of a $z$-axis MEMS gyroscope.

angular velocity is almost constant over a long enough time interval, the equation of motion of a gyroscope is simplified as follows.

Taking fabrication imperfections, which cause extra coupling between $x$ - and $y$-axes, and external disturbances into account, the governing equation for MEMS gyroscope is

$$
\begin{aligned}
& m \ddot{x}+d_{x x} \dot{x}+d_{x y} \dot{y}+k_{x x} x+k_{x y} y=u_{x}+2 m \Omega_{z} \dot{y}, \\
& m \ddot{y}+d_{x y} \dot{x}+d_{y y} \dot{y}+k_{x y} x+k_{y y} y=u_{y}-2 m \Omega_{z} \dot{x} .
\end{aligned}
$$

In (1) $d_{x x}$ and $d_{y y}$ are damping; $k_{x x}$ and $k_{y y}$ are spring coefficients; $d_{x y}$ and $k_{x y}$ called quadrature errors are coupled damping and spring terms, respectively, due in large part to the asymmetries in suspension structure and misalignment of sensors and actuators.

Dividing both sides of (1) by $m, q_{0}$, and $\omega_{0}^{2}$, which are a reference mass, length, and natural or resonant frequency, respectively, and considering lumped external disturbances, we get the form of the nondimensional equation of motion as

$$
\begin{aligned}
& \ddot{x}+d_{x x} \dot{x}+d_{x y} \dot{y}+\omega_{x}^{2} x+\omega_{x y} y=u_{x}+2 \Omega_{z} \dot{y}+d_{1}, \\
& \ddot{y}+d_{x y} \dot{x}+d_{y y} \dot{y}+\omega_{x y} x+\omega_{y}^{2} y=u_{y}-2 \Omega_{z} \dot{x}+d_{2},
\end{aligned}
$$

where $d_{x x} / m \omega_{0} \rightarrow d_{x x}, d_{x y} / m \omega_{0} \rightarrow d_{x y}, d_{y y} / m \omega_{0} \rightarrow d_{y x y}$, $\Omega_{z} / \omega_{0} \rightarrow \Omega_{z}, \sqrt{k_{x x} / m \omega_{0}^{2}} \rightarrow \omega_{x}, \sqrt{k_{y y} / m \omega_{0}^{2}} \rightarrow \omega_{y}$, and $k_{x y} / m \omega_{0}^{2} \rightarrow \omega_{x y}$, and $d_{1}$ and $d_{2}$ denote disturbances in the two axes. Note that, the lumped disturbances $d_{1}$ and $d_{2}$ could also contain the effects of the time-varying unknown but bounded parameter uncertainties.

The vector form of MEMS gyroscope dynamics model can be written as

$$
\ddot{q}+D \dot{q}+K_{b} q=u-2 \Omega \dot{q}+f
$$

where $q=\left[\begin{array}{l}x \\ y\end{array}\right], u=\left[\begin{array}{l}u_{x} \\ u_{y}\end{array}\right], f=\left[\begin{array}{l}d_{1} \\ d_{2}\end{array}\right], \Omega=\left[\begin{array}{cc}0 & -\Omega_{z} \\ \Omega_{z} & 0\end{array}\right], D=$ $\left[\begin{array}{ll}d_{x x} & d_{x y} \\ d_{x y} & d_{y y}\end{array}\right]$, and $K_{b}=\left[\begin{array}{cc}\omega_{x}^{2} & \omega_{x y} \\ \omega_{x y} & \omega_{y}^{2}\end{array}\right]$, and $f$ represents the matched lumped parameter uncertainty and external disturbance. 
We make the following assumption: the lumped uncertainty and disturbance $f$ is bounded such that $\|f\| \leq \alpha$, where $\alpha$ is a known positive constant.

\section{Robust Adaptive Control}

In this section, a robust adaptive control scheme with application to a MEMS gyroscope is given. The control objective is to improve the robustness of the control system and minimize system nonlinearities; thus all unknown system parameters and angular velocity can be estimated correctly. The block diagram of a robust adaptive control for a MEMS gyroscope is shown in Figure 2. The tracking error between gyroscope state and reference state acts as the input of the robust adaptive controller which is proposed to control the system; thus the angular velocity and all the unknown parameters can be estimated.

Suppose that a reference trajectory is generated by an ideal oscillator and the control target is to make the trajectory of the gyroscopes follow that of the reference model. The reference model $x_{m}=A_{1} \sin \left(w_{1} t\right)$ and $y_{m}=A_{2} \sin \left(w_{2} t\right)$ can be redefined in vector terms as

$$
\ddot{q}_{m}+k_{m} q_{m}=0
$$

where $q=\left[\begin{array}{l}q_{m 1} \\ q_{m 2}\end{array}\right], k_{m}=\left[\begin{array}{cc}w_{1}^{2} & 0 \\ 0 & w_{2}^{2}\end{array}\right] \cdot e=q-q_{m}$ is the tracking error.

The sliding surface is designed as

$$
s=\dot{e}+c e,
$$

where $c$ is a positive definite constant matrix which needs to be selected, $c=\operatorname{diag}\left\{\lambda_{1}, \lambda_{2}\right\}$.

The derivative of the sliding surface is

$$
\dot{s}=u+f-(D+2 \Omega) \dot{q}-K q+c\left(\dot{q}-\dot{q}_{m}\right)+K_{m} q_{m} .
$$

Substituting $D=\left[\begin{array}{ll}d_{y y} & d_{x y} \\ d_{x y} & d_{y y}\end{array}\right], K=\left[\begin{array}{cc}w_{x}^{2} & w_{x y} \\ w_{x y} & w_{y}^{2}\end{array}\right]$, and $\Omega=\left[\begin{array}{cc}0 & -\Omega_{z} \\ \Omega_{z} & 0\end{array}\right]$ into (4) yields

$$
\begin{aligned}
\dot{s}= & u+f-\left[\begin{array}{cc}
d_{x x} & d_{x y}-2 \Omega_{z} \\
d_{x y}+2 \Omega_{z} & d_{y y}
\end{array}\right]\left[\begin{array}{l}
q_{1} \\
q_{2}
\end{array}\right] \\
& -\left[\begin{array}{cc}
w_{x}^{2} & w_{x y} \\
w_{x y} & w_{y}^{2}
\end{array}\right]\left[\begin{array}{l}
q_{1} \\
q_{2}
\end{array}\right]+c\left(\dot{q}-\dot{q}_{m}\right)+K_{m} q_{m} .
\end{aligned}
$$

Rewriting (5) yields

$$
\dot{s}=u+f-\left[\begin{array}{ccccccc}
\dot{q}_{1} & \dot{q}_{2} & 0 & -2 q_{2} & q_{1} & q_{2} & 0 \\
0 & \dot{q}_{1} & \dot{q}_{2} & 2 q_{1} & 0 & q_{1} & q_{2}
\end{array}\right]\left[\begin{array}{c}
d_{x x} \\
d_{x y} \\
d_{y y} \\
\Omega_{z} \\
w_{x}^{2} \\
w_{x y} \\
w_{y}^{2}
\end{array}\right]
$$

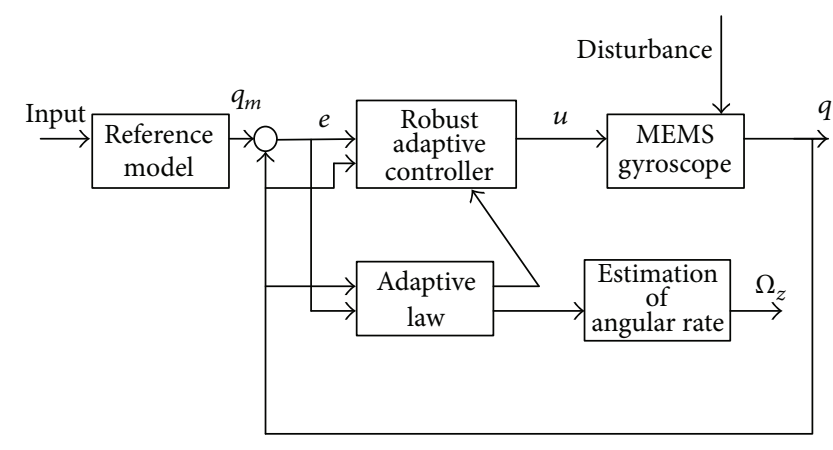

FIGURE 2: Block diagram of a robust adaptive control for a MEMS gyroscope.

Define

$$
\begin{gathered}
Y=\left[\begin{array}{ccccccc}
\dot{q}_{1} & \dot{q}_{2} & 0 & -2 q_{2} & q_{1} & q_{2} & 0 \\
0 & \dot{q}_{1} & \dot{q}_{2} & 2 \dot{q}_{1} & 0 & q_{1} & q_{2}
\end{array}\right], \\
\theta^{*}=\left[\begin{array}{ccccccc}
d_{x x} & d_{x y} & d_{y y} & \Omega_{z} & w_{x}^{2} & w_{x y} & w_{y}^{2}
\end{array}\right]^{T}, \\
Q=c\left(\dot{q}-\dot{q}_{m}\right)+K_{m} q_{m} .
\end{gathered}
$$

Then, (6) becomes

$$
\dot{s}=u+f-Y \theta^{*}+Q,
$$

where $Y\left(q_{1}, \dot{q}_{1}, q_{2}, \dot{q}_{2}\right)$ is a $2 \times 7$ matrix of known functions and $\theta^{*}$ contains unknown system parameters. We assume both position and velocity are measurable.

Setting $\dot{s}=0$ to solve equivalent control $u_{\text {eq }}$ gives

$$
u_{\text {eq }}=Y \theta^{*}-Q-f \text {. }
$$

The adaptive controller $u$ is proposed as

$$
u=Y \theta-Q+u_{s 1}+u_{s 2}=Y \theta-Q-K_{s} s-\rho \frac{s}{\|s\|},
$$

where $u=\left[\begin{array}{l}u_{1} \\ u_{2}\end{array}\right], s=\left[\begin{array}{l}s_{1} \\ s_{2}\end{array}\right], K_{s}=\left[\begin{array}{cc}k_{s 1} & 0 \\ 0 & k_{s 2}\end{array}\right]>0, \rho=\left[\begin{array}{cc}\rho_{1} & 0 \\ 0 & \rho_{2}\end{array}\right]>$ 0 , and $K_{s}$ and $\rho$ are constant matrices to satisfy the desired transient performance of the control system. $\theta$ is the estimate of $\theta^{*} ; u_{s 1}=-k_{s} s$ is a feedback component. Consider

$$
u_{s 2}=\left[\begin{array}{l}
u_{s 21} \\
u_{s 22}
\end{array}\right]=-\rho \frac{s}{\|s\|}=-\left(\begin{array}{cc}
\rho_{1} & 0 \\
0 & \rho_{2}
\end{array}\right)\left(\begin{array}{c}
\frac{s_{1}}{\sqrt{s_{1}^{2}+s_{2}^{2}}} \\
\frac{s_{2}}{\sqrt{s_{1}^{2}+s_{2}^{2}}}
\end{array}\right)
$$

is the sliding mode control component.

Substituting (12) into (10) yields

$$
\dot{s}=Y \widetilde{\theta}-Q-K_{s} s-\rho \frac{s}{|s|},
$$

where $\widetilde{\theta}=\theta-\theta^{*}$.

Consider the following Lyapunov function:

$$
V=\frac{1}{2} s^{T} P s+\frac{1}{2} \widetilde{\theta}^{T} m^{-1} \tilde{\theta},
$$

where $P=P^{T}, m=m^{T}$ are positive definite and symmetric matrix. 


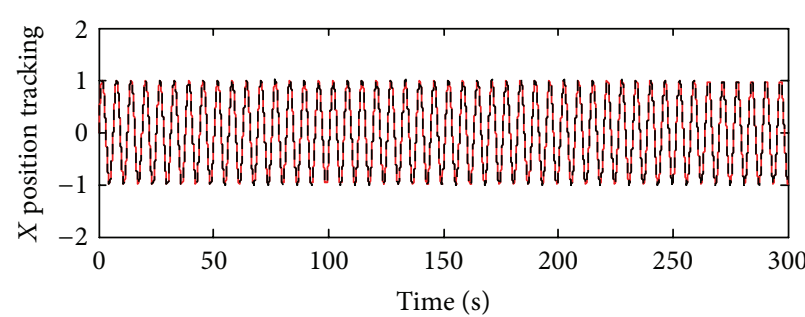

_ Ideal position signal - - - $X$ position tracking

(a)

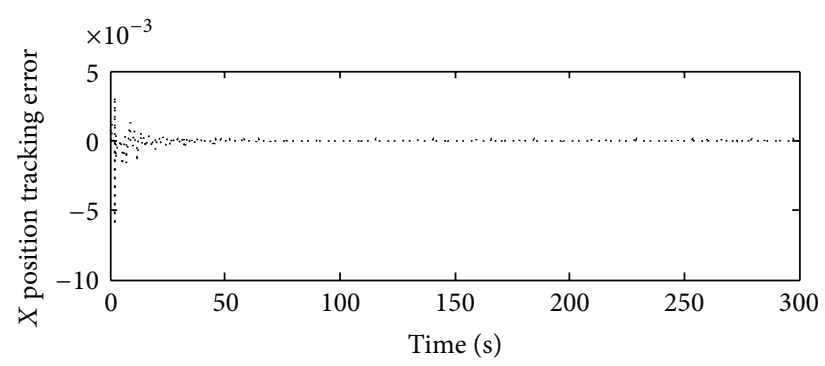

(c)

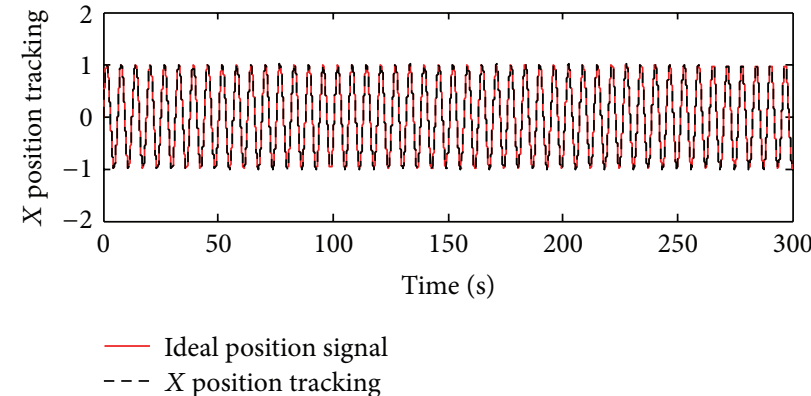

(b)

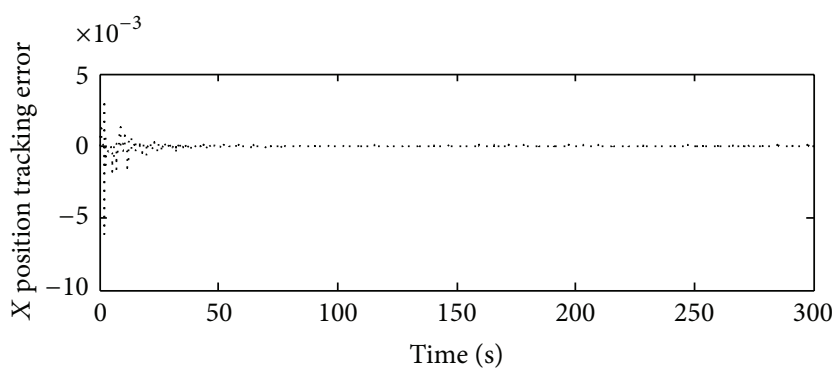

(d)

FIgURE 3: Tracking error using robust adaptive control.

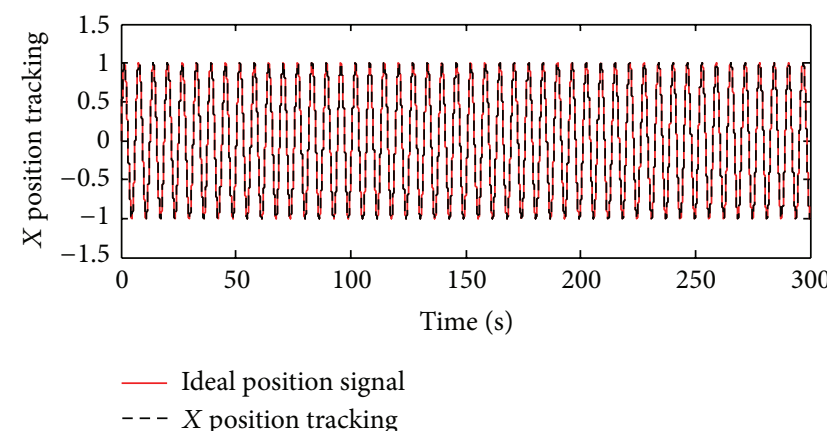

(a)

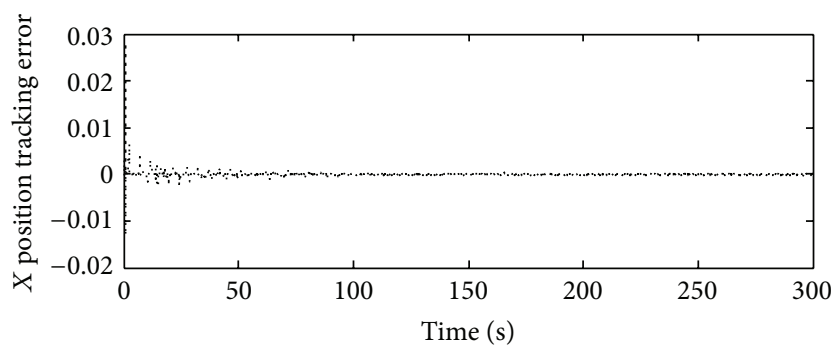

(c)

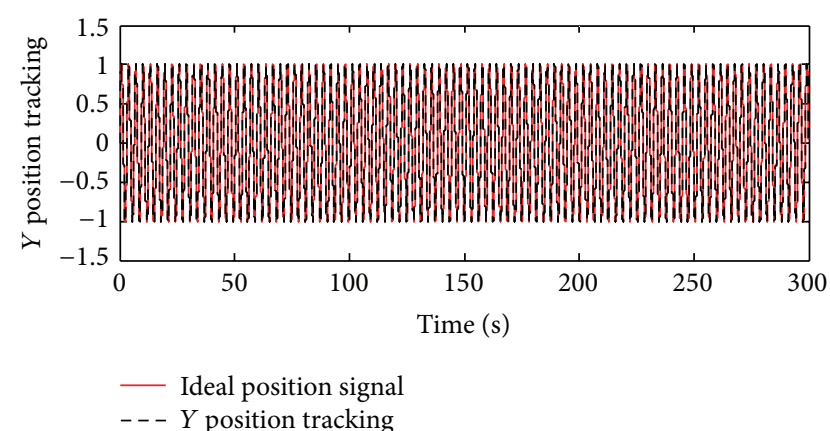

(b)

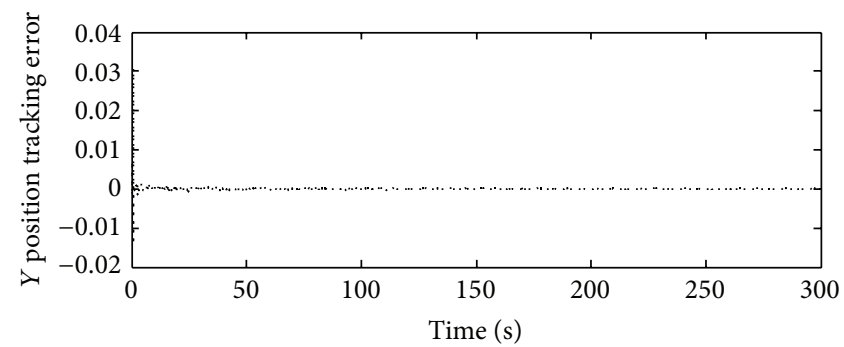

(d)

FIGURE 4: Tracking error using standard adaptive control.

The derivative of $V$ is

$$
\begin{aligned}
\dot{V} & =s^{T} P \dot{s}+\dot{\tilde{\theta}}^{T} m^{-1} \tilde{\theta} \\
& =s^{T} P\left(Y \tilde{\theta}+f-\rho \frac{s}{\|s\|}-K_{s} s\right)+\dot{\tilde{\theta}}^{T} m^{-1} \tilde{\theta} \\
& =s^{T} P f-P \rho|s|-s^{T} P K_{s} s+\left(s^{T} P Y \widetilde{\theta}+\dot{\tilde{\theta}}^{T} m^{-1} \tilde{\theta}\right) .
\end{aligned}
$$

To make $\dot{V} \leq 0$, choose the robust adaptive law as

$$
\dot{\theta}=-m Y^{T} P s .
$$

The choice yields

$$
\begin{aligned}
\dot{V} & =s^{T} P f-P \rho|s|-s^{T} P K_{s} s \\
& \leq|s| P|f|-P \rho|s|-s^{T} P K_{s} s
\end{aligned}
$$




$$
=-|s| P(\rho-|f|)-s^{T} P K_{s} s .
$$

In (18), the second term represents the effect of the feedback component $u_{s 1}=-k_{s} s$. Without the feedback component and with $\rho$ satisfying $\rho \geq \alpha+\eta$, where $\eta$ is a positive constant, $\dot{V}$ becomes negative semidefinite; that is, $\dot{V} \leq$ $-\eta\|s\| \leq 0 . \dot{V}$ is negative definite implying that $s$ and $\widetilde{K}$ converge to zero. $\dot{V}$ is negative semidefinite ensuring that $V$, $s$, and $\widetilde{\theta}$ are all bounded. It can be concluded from (14) that $\dot{s}$ is also bounded. Barbalat's lemma can be used to prove that $\lim _{t \rightarrow \infty} s(t)=0$. The inequality $\dot{V} \leq-\eta\|s\|$ implies that $s$ is integrable as $\int_{0}^{t}\|s\| d t \leq(1 / \eta)[V(0)-V(t)]$. Since $V(0)$ is bounded and $V(t)$ is nonincreasing and bounded, it can be concluded that $\lim _{t \rightarrow \infty} \int_{0}^{t}\|s\| d t$ is bounded. Since $\lim _{t \rightarrow \infty} \int_{0}^{t}\|s\| d t$ is bounded and $\dot{s}$ is also bounded, according to Barbalat's lemma, $s(t)$ will asymptotically converge to zero, $\lim _{t \rightarrow \infty} s(t)=0$. If $Y$ is persistently exciting signal [17], then $\dot{\tilde{\theta}}(t)=-\tau Y^{T} s(t)$ guarantees that $\tilde{\theta} \rightarrow 0, \theta$ will converge to its true values. Then the unknown angular velocity as well as all other unknown parameters can be consistently estimated and the control is asymptotically stable.

Taking the feedback component into account, $\dot{V} \leq$ $-s^{T} P K_{s} s \leq-\gamma_{\min }\left(P K_{s}\right)\|s\|^{2}$, where $\gamma_{\text {min }}\left(P K_{s}\right)$ is the minimum eigenvalue $P K_{s}$ and $\gamma_{\min }\left(P K_{s}\right)\|s\|^{2} \in \kappa$ based on Lemma 1. In (15), $V=(1 / 2) s^{T} P s+(1 / 2) \widetilde{\theta}^{T} m^{-1} \tilde{\theta}=(1 / 2) \Theta^{T}\left[\begin{array}{cc}P & 0 \\ 0 & m^{-1}\end{array}\right] \Theta$, where $\Theta=\left[\begin{array}{ll}s^{T} & \widetilde{\theta}^{T}\end{array}\right]^{T}$. Therefore $(1 / 2) \lambda_{\min }(M)\|\Theta\|^{2} \leq V=$ $(1 / 2) \Theta^{T} M \Theta \leq(1 / 2) \lambda_{\max }(M)\|\Theta\|^{2}$, where $M=\left[\begin{array}{cc}P & 0 \\ 0 & m^{-1}\end{array}\right]$. Because $(1 / 2) \lambda_{\text {min }}(M)\|\Theta\|^{2} \in \kappa$ and $(1 / 2) \lambda_{\text {max }}(M)\|\Theta\|^{2} \in \kappa$ based on Lemma 1 and according to Lemma 2 the closed-loop system is exponentially stable. Therefore incorporating the feedback component into the standard adaptive control could improve the stability of the control system better.

Lemma 1. A continuous function $\varphi:\left[\begin{array}{ll}0 & r\end{array}\right] \rightarrow R^{+}$(or a continuous function $\varphi:\left[\begin{array}{ll}0 & \infty\end{array}\right] \rightarrow R^{+}$) is said to belong to Class $\kappa$; that is, $\varphi \in \kappa$ if

(1) $\varphi(0)=0$,

(2) $\varphi$ is strictly increasing on $\left[\begin{array}{ll}0 & r\end{array}\right]$ (or on $\left[\begin{array}{ll}0 & \infty\end{array}\right]$ ).

Lemma 2. If $V$ is decrescent and there exist $\varphi_{1}, \varphi_{2}, \varphi_{3} \in \kappa$ of the same order of magnitude such that

$$
\varphi_{1}(|x|) \leq V(t, x) \leq \varphi_{2}(|x|), \quad \dot{V}(t, x) \leq-\varphi_{3}(|x|)
$$

for all $x \in \beta(r)$ and $t \in R^{+}$, then $x_{e}=0$ is exponentially stable.

Remark 3. For the purpose of comparison, a standard adaptive control is also proposed for a MEMS gyroscope. The difference between robust adaptive control and standard adaptive control schemes is that a feedback controller is not incorporated into the adaptive controller which is proposed as

$$
u=Y \theta-Q-\rho \frac{s}{\|s\|}
$$

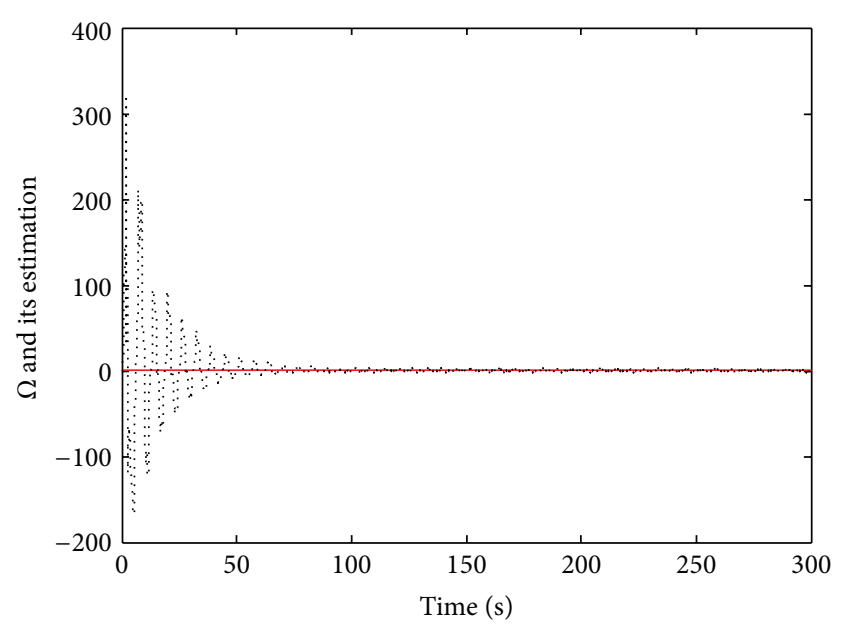

FiguRE 5: Adaptation of angular velocity using robust control.

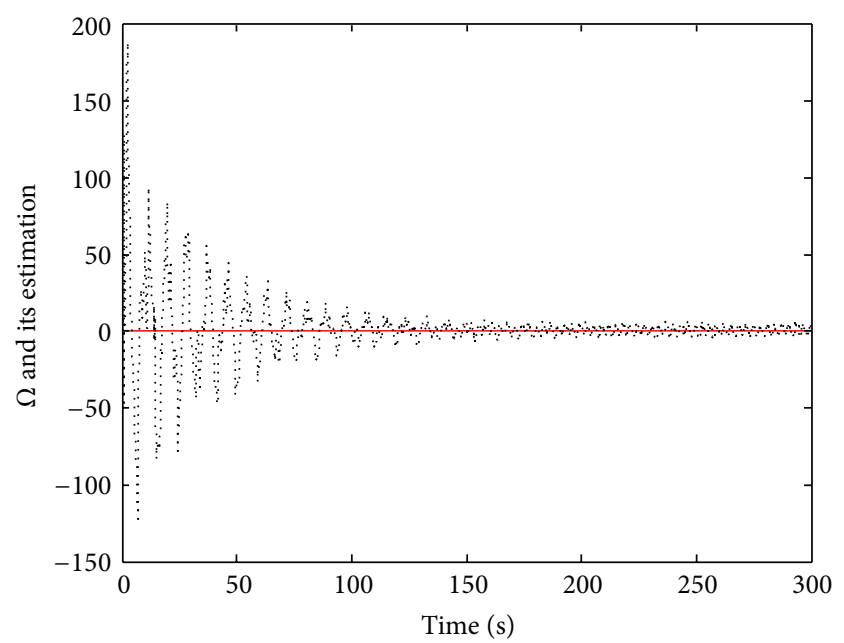

FIgURE 6: Adaptation of angular velocity using standard adaptive control.

Remark 4. In order to eliminate the control discontinuities, a smooth sliding mode control that can reduce chattering problem is proposed as

$$
u=Y \theta-Q-K_{s} s-\rho \frac{s}{\|s\|+\varepsilon},
$$

where $\varepsilon$ is a small positive constant.

\section{Simulation Example}

In this section, the robust adaptive sliding control and the adaptive sliding control are both evaluated on the MEMS gyroscope model for the purpose of comparison. The control objective is to estimate angular velocity and all the unknown parameters and make the control system track the reference trajectory. Random signal with zero mean and unity variance 


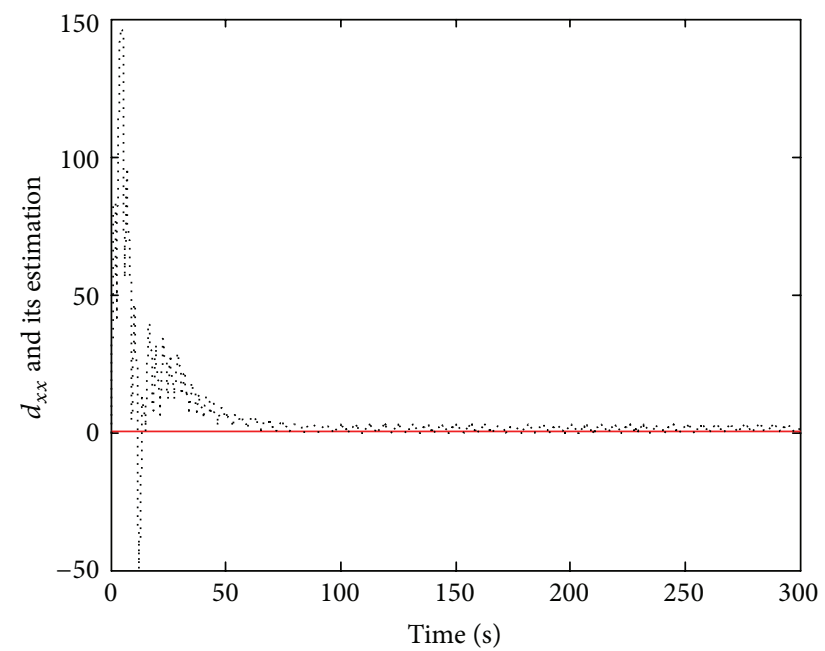

(a)

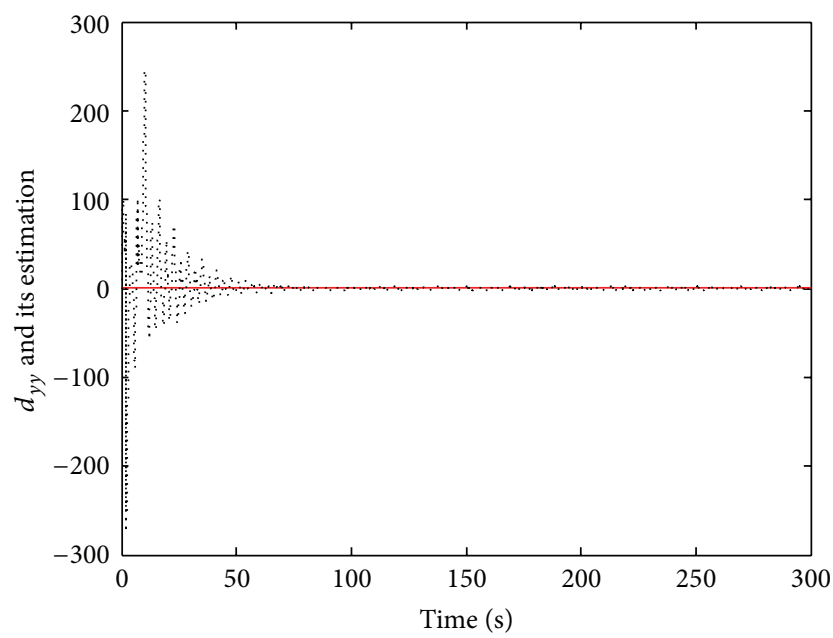

(c)

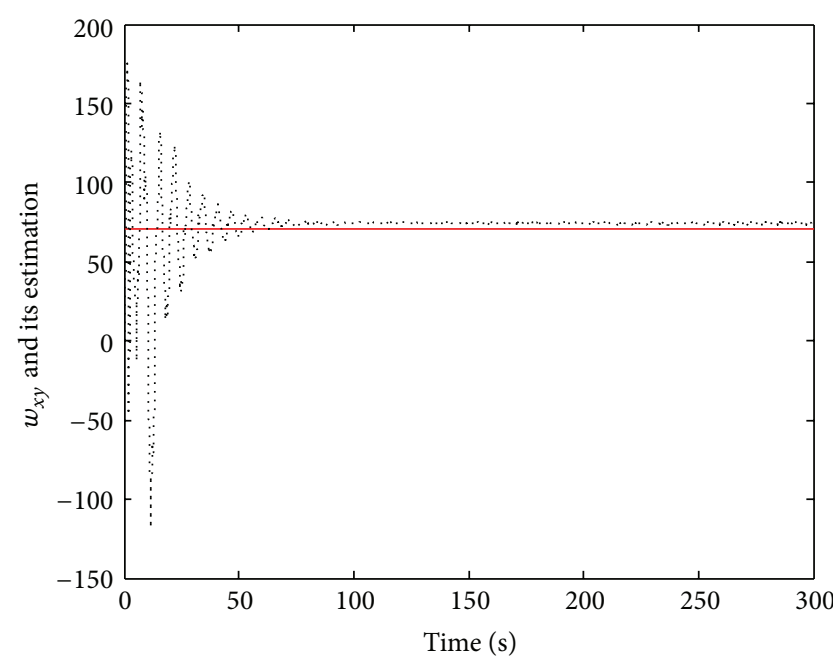

(e)

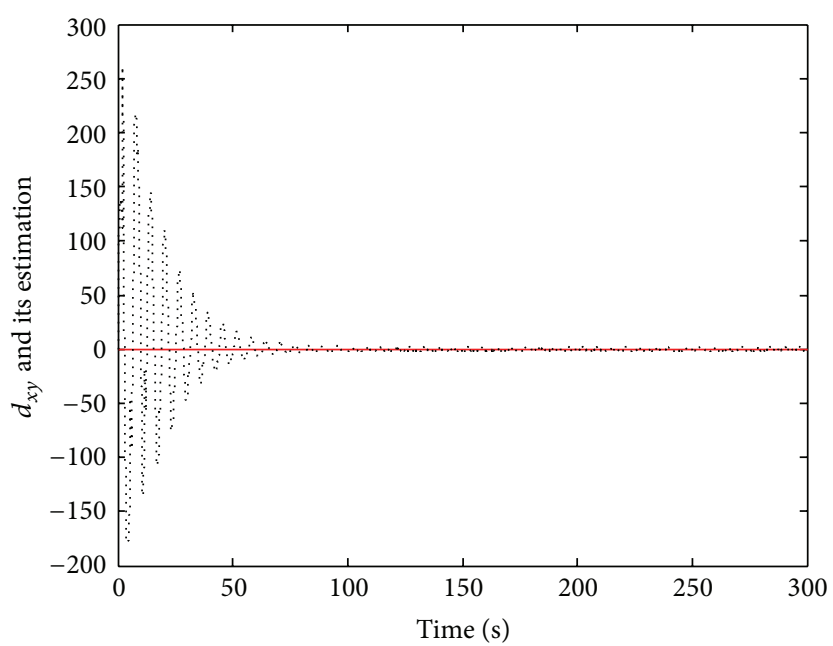

(b)

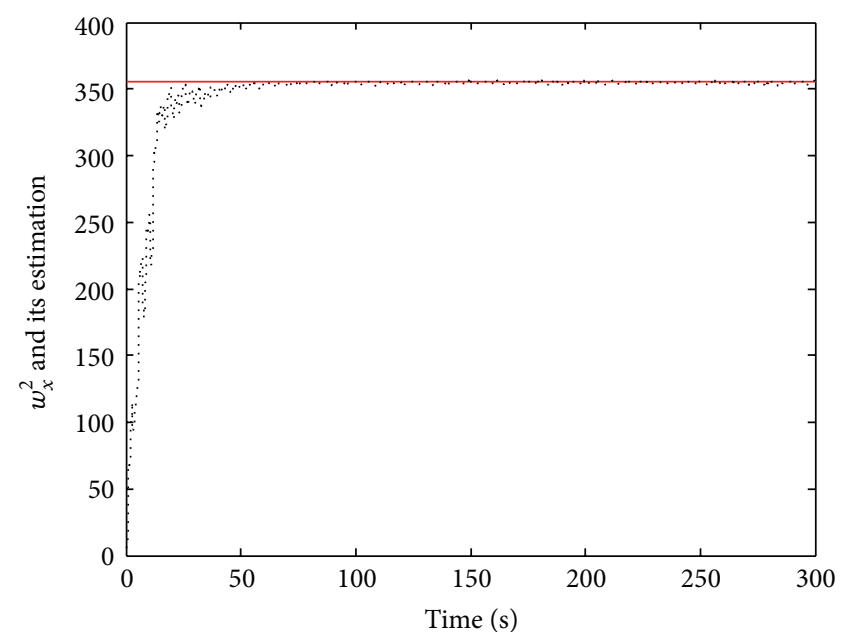

(d)

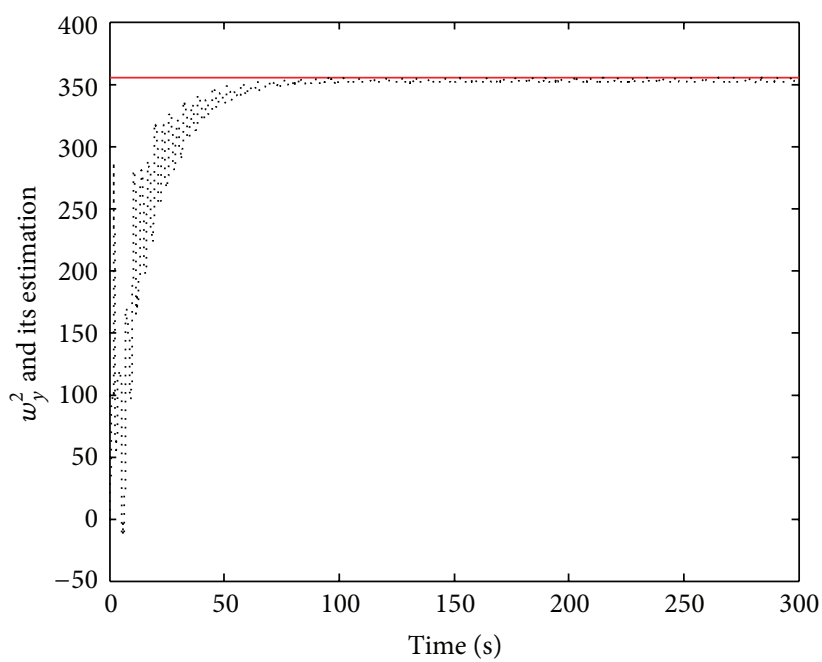

(f)

Figure 7: Adaptation of control parameters using robust adaptive control. 


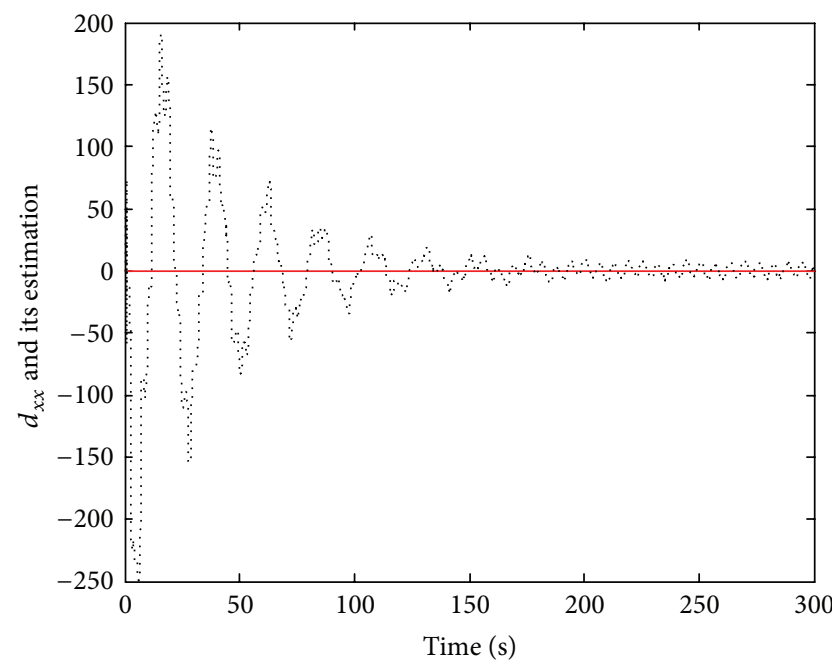

(a)

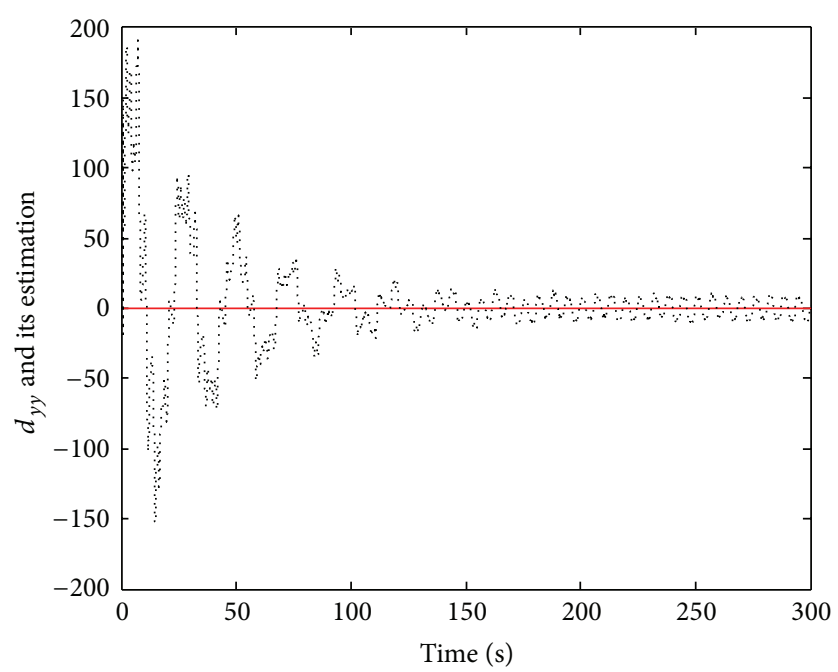

(c)

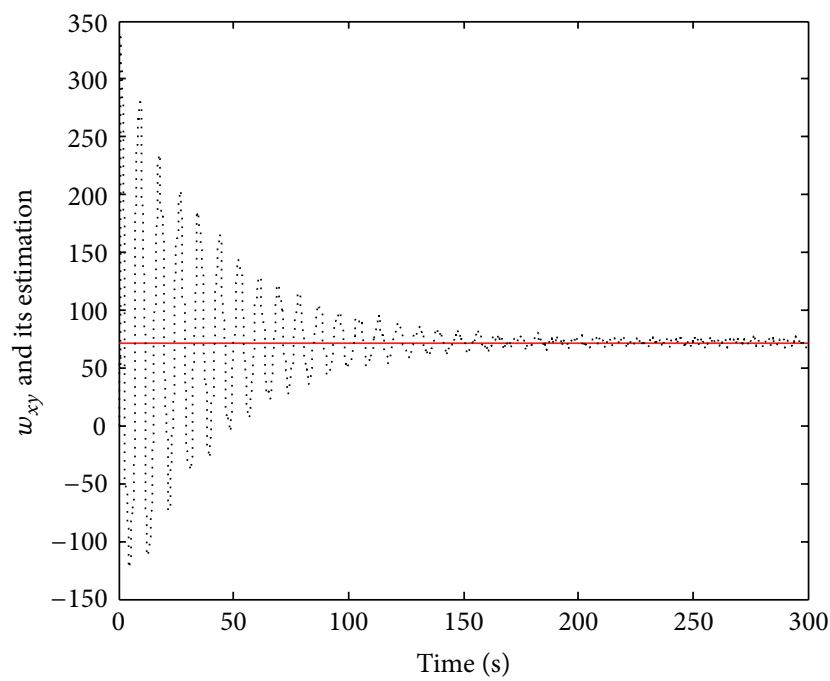

(e)

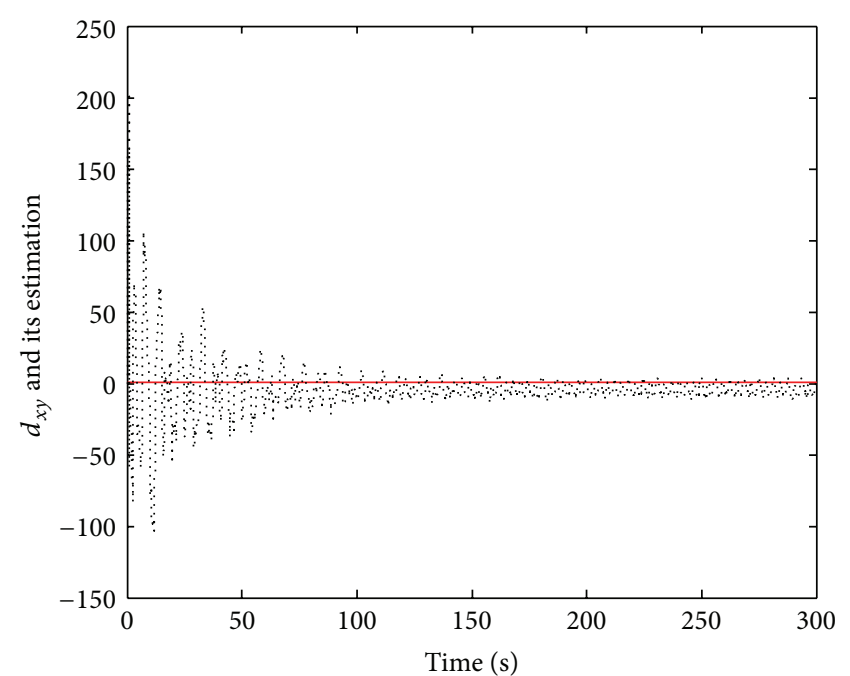

(b)

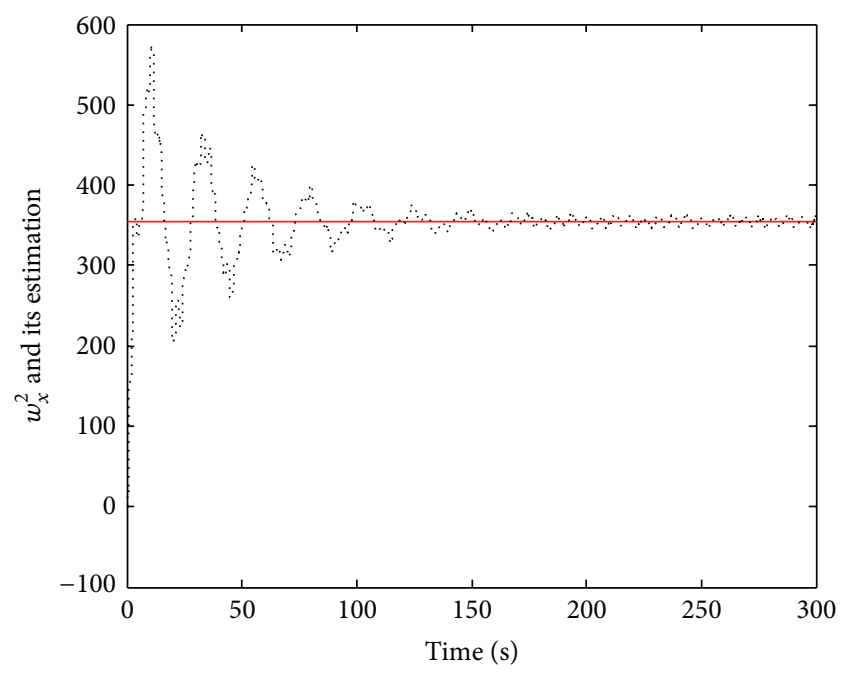

(d)

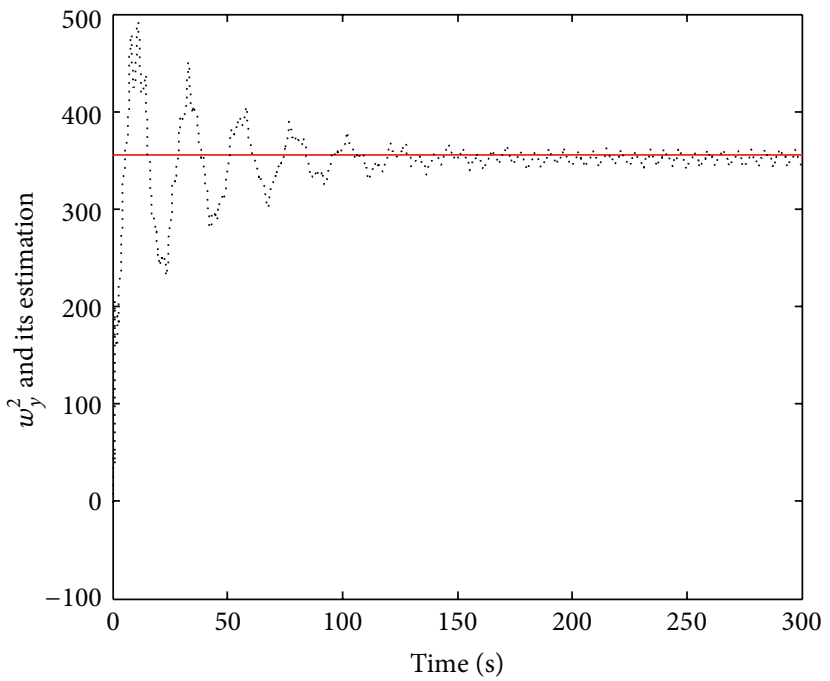

(f)

FIGURE 8: Adaptation of control parameters using standard adaptive control. 


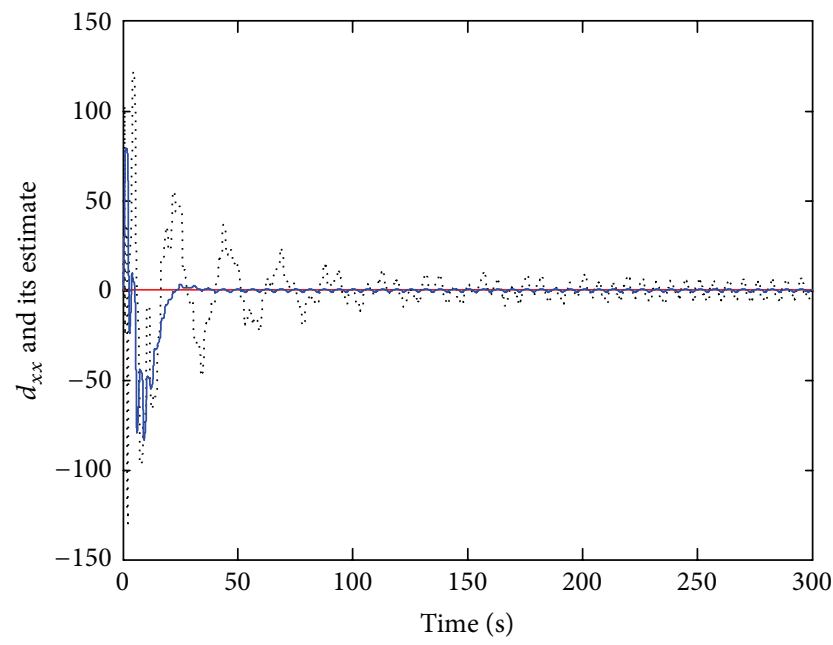

(a)

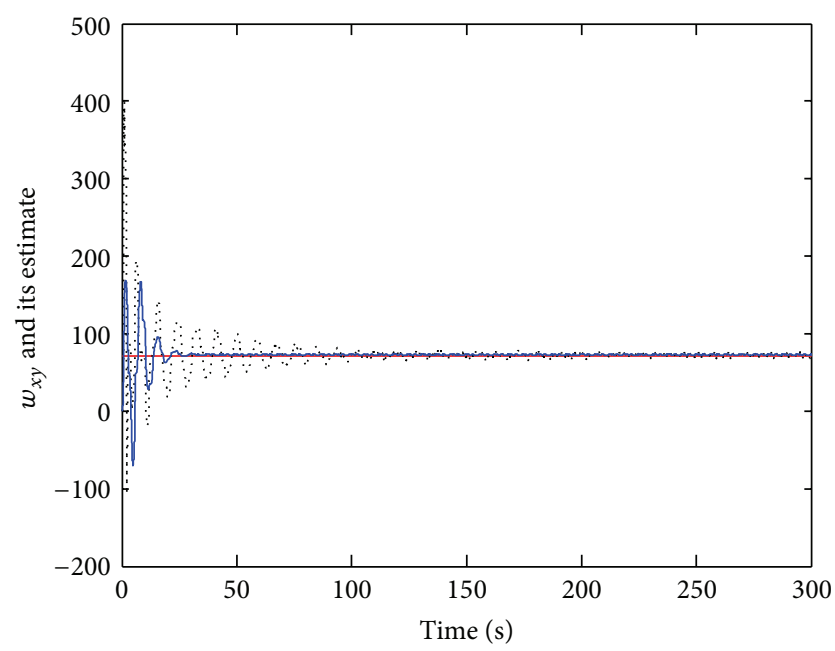

(c)

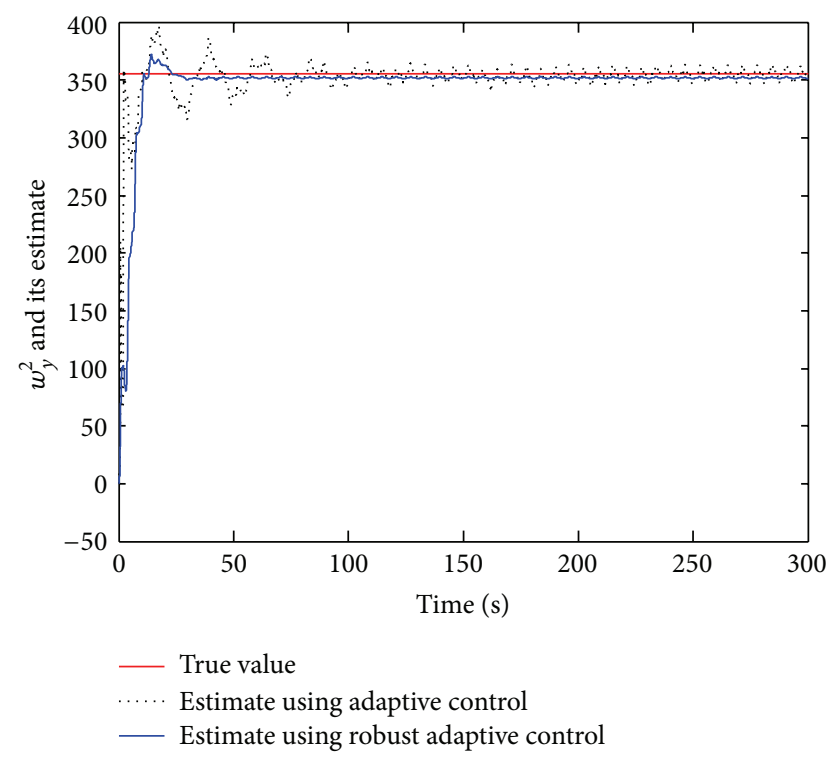

(e)

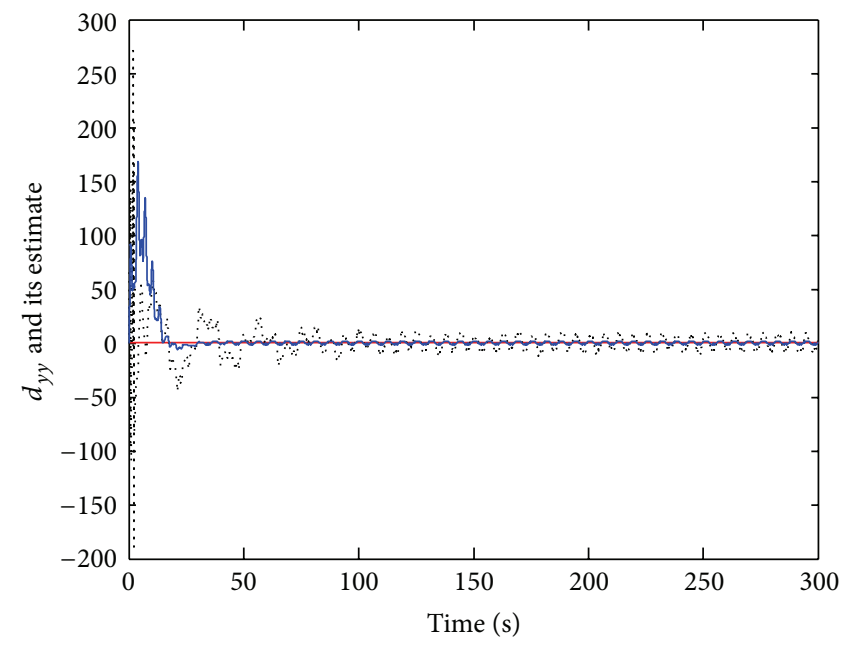

(b)

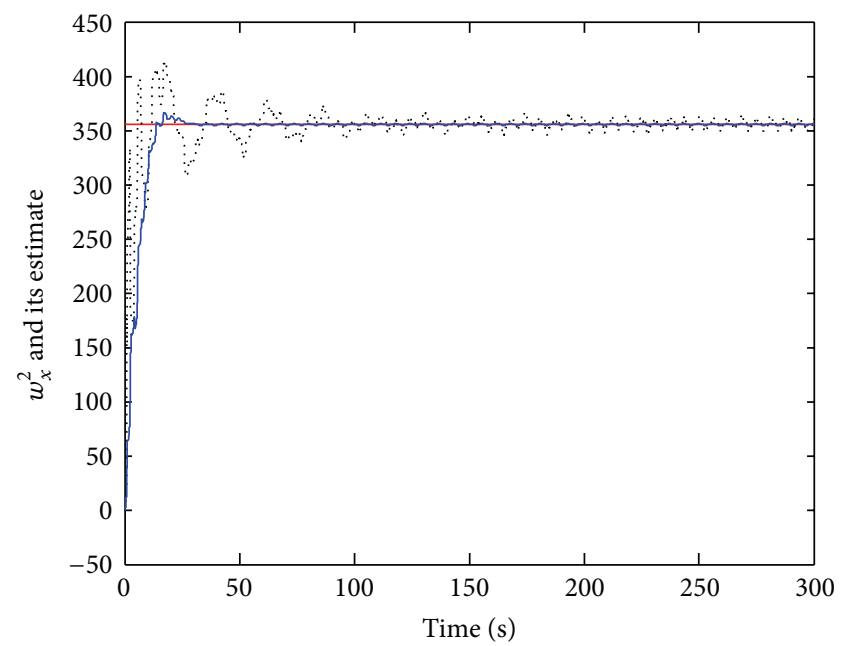

(d)

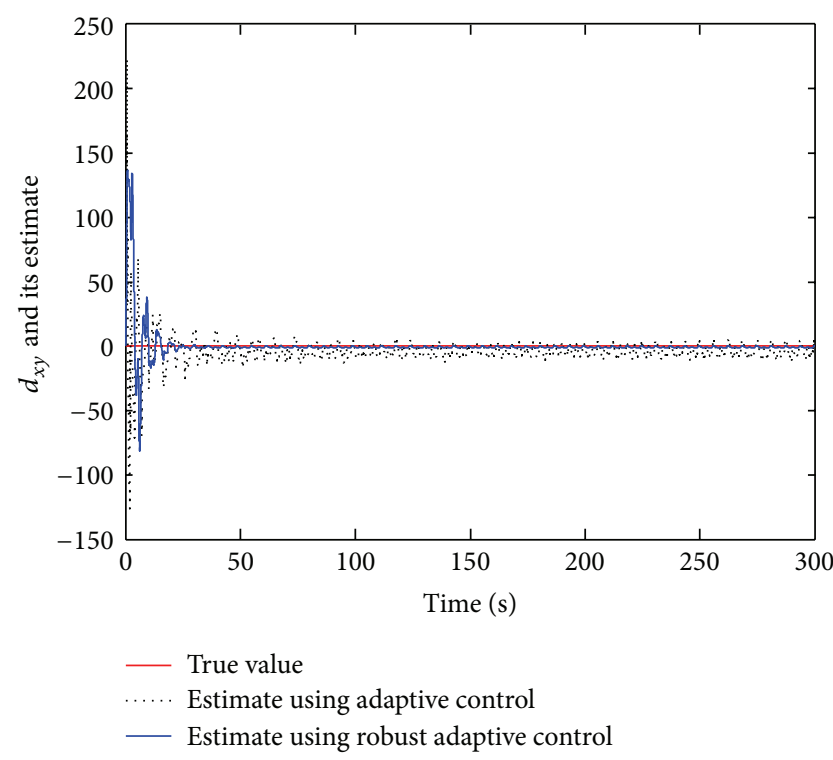

(f)

FIGURE 9: Comparison of system parameters between robust adaptive control and adaptive control. 
is considered as external disturbance. The parameters of the MEMS gyroscope are listed as follows:

$$
\begin{gathered}
m=1.8 \times 10^{-7} \mathrm{~kg}, \quad k_{x x}=63.955 \mathrm{~N} / \mathrm{m}, \\
k_{y y}=95.92 \mathrm{~N} / \mathrm{m}, \quad k_{x y}=12.779 \mathrm{~N} / \mathrm{m}, \\
d_{x x}=1.8 \times 10^{-6} \mathrm{~N} \cdot \mathrm{s} / \mathrm{m}, \quad d_{y y}=1.8 \times 10^{-6} \mathrm{~N} \cdot \mathrm{s} / \mathrm{m}, \\
d_{x y}=3.6 \times 10^{-7} \mathrm{~N} \cdot \mathrm{s} / \mathrm{m} .
\end{gathered}
$$

Choose 1 micrometer as the reference length $q_{0}$ and $1 \mathrm{kHz}$ as natural frequency of each axis of MEMS gyroscopes. The unknown angular velocity is assumed as $\Omega_{z}=5.0 \mathrm{rad} / \mathrm{s}$. The nondimensional parameters are calculated as follows:

$$
\begin{gathered}
w_{x}^{2}=355.3, \quad w_{y}^{2}=532.9, \\
w_{x y}=70.99, \quad d_{x x}=0.01, \\
d_{y y}=0.01, \quad d_{x y}=0.002, \quad \Omega_{z}=0.1 .
\end{gathered}
$$

The desired motion trajectories are $x_{m}=A_{1} \sin \left(w_{1} t\right), y_{m}=$ $A_{2} \sin \left(w_{2} t\right)$, where $w_{1}=1 \mathrm{kHz}, w_{2}=2 \mathrm{kHz}$. The $m$ and $P$ in (17) are chosen as $m=\operatorname{diag}\{10,10,10,10,10,10,10\}$ and $P=\operatorname{diag}\{1000,1000\}$. The $K_{s}$ and $\rho$ in (12) are chosen as $K_{s}=$ $\operatorname{diag}\{1000,1000\}$ and $\rho=\operatorname{diag}\{100,100\}$.

Figures 3 and 4 compare the tracking errors, where $e_{1}=$ $x-x_{m}$ denotes tracking error in $x$ axle and $e_{2}=y-y_{m}$ denotes tracking error in $y$ axle. It is observed that the tracking errors all converge to zero asymptotically and the tracking error of robust adaptive sliding control has better transient performance than that of standard adaptive sliding control. Figures 5 and 6 compare the adaptation of angular velocity; it can be seen that the estimation of angular velocity $\Omega_{z}$ using the robust adaptive control converges to its true value more quickly than that using standard adaptive sliding control. Figures 7, 8, and 9 compare the adaptation of parameters using these two different controllers, respectively. It can be observed from these figures that the former achieves better parameter identification performance than the latter.

In summary, with the control law (12) and the parameter adaptation law (17), if the gyroscope is controlled to follow the mode-unmatched reference model, the persistent excitation condition is satisfied; that is, $w_{1} \neq w_{2}$, and all unknown gyroscope parameters, including the angular velocity, are estimated correctly. In the presence of external disturbance and model uncertainties, the robust adaptive sliding control has better robustness and transient dynamic characteristics than adaptive sliding control.

\section{Conclusion}

In the paper, a novel robust adaptive control scheme is proposed for MEMS gyroscopes. For the purpose of comparison, a robust adaptive control and a standard adaptive control without feedback algorithm are investigated. Numerical simulations show that if the persistent excitation can be satisfied, all unknown parameters, including the angular velocity, converge to their true values, and tracking error converges to zero asymptotically, using these two adaptive approaches. But in the presence of external disturbance and model uncertainties, the robust adaptive control has better robustness and dynamic characteristics than standard adaptive control.

\section{Acknowledgments}

The authors thank the anonymous reviewers for useful comments that improved the quality of the paper. This work is partially supported by National Science Foundation of China under Grant no. 61374100; Natural Science Foundation of Jiangsu Province under Grant no. BK20131136; the Fundamental Research Funds for the Central Universities under Grant no. 2012B06714.

\section{References}

[1] N. O. P. Arancibia, N. Chen, S. Gibson, and T.-C. Tsao, "Adaptive control of a MEMS steering mirror for suppression of laser beam jitter," in Proceedings of the American Control Conference (ACC '05), pp. 3586-3591, June 2005.

[2] J. Fei and J. Zhou, "Robust adaptive control of MEMS triaxial gyroscope using fuzzy compensator," IEEE Transactions on Systems, Man, and Cybernetics, Part B, vol. 42, no. 6, pp. 15991607, 2012.

[3] J. Fei and C. Batur, "Robust adaptive control for a MEMS vibratory gyroscope," International Journal of Advanced Manufacturing Technology, vol. 42, no. 3-4, pp. 293-300, 2009.

[4] S. Jagannathan and M. Hameed, "Adaptive force-balancing control of MEMS gyroscope with actuator limits," in Proceedings of the American Control Conference (AAC '04), vol. 2, pp. 18621867, July 2004.

[5] G. Zhu, J. Lévine, L. Praly, and Y.-A. Peter, "Flatness-based control of electrostatically actuated MEMS with application to adaptive optics: a simulation study," Journal of Microelectromechanical Systems, vol. 15, no. 5, pp. 1165-1174, 2006.

[6] R. P. Leland, "Adaptive control of a MEMS gyroscope using lyapunov methods," IEEE Transactions on Control Systems Technology, vol. 14, no. 2, pp. 278-283, 2006.

[7] S. Park and R. Horowitz, "Adaptive control for the conventional mode of operation of MEMS gyroscopes," Journal of Microelectromechanical Systems, vol. 12, no. 1, pp. 101-108, 2003.

[8] C. Wang, S. Wang, and Y. Yin, "A dual-mass MEMS vibratory gyroscope with adaptive control scheme," in Proceedings of the 7 th IEEE International Conference on Nanotechnology (NANO '07), pp. 25-28, August 2007.

[9] D. Liu, N. N. Lu, J. Cui et al., "Digital closed-loop control based on adaptive filter for drive mode of a MEMS gyroscope," in Proceedings of the 9th IEEE Sensors Conference (SENSORS '10), pp. 1722-1726, November 2010.

[10] M. H. Salah, M. L. McIntyre, D. M. Dawson, J. R. Wagner, and E. Tatlicioglu, "Sensing of the time-varying angular rate for MEMS Z-axis gyroscopes," Mechatronics, vol. 20, no. 6, pp. 720-727, 2010.

[11] W. Wang, X. Lu, and F. Sun, "Design of micromachined vibratory gyroscope with two degree-of-freedom drive-mode and sense-mode," IEEE Sensors Journal, vol. 12, no. 7, pp. 2460-2464, 2012.

[12] C. Tsai, K. Chen, and C. Shen J, "Tsai A MEMS doubly decoupled gyroscope with wide driving frequency range," IEEE 
Transactions on Industrial Electronics, vol. 59, no. 12, pp. 49214929, 2012.

[13] H. Chang, L. Xue, and C. Jiang, "Combining numerous uncorrelated MEMS gyroscopes for accuracy improvement based on an optimal kalman filter," IEEE Transactions on Instrumentation and Measurement, vol. 61, no. 11, pp. 3084-3093, 2012.

[14] M. Egretzberger, F. Mair, and A. Kugi, "Model-based control concepts for vibratory MEMS gyroscopes," Mechatronics, vol. 22, no. 3, pp. 241-250, 2012.

[15] G. Ma, W. Chen, W. Zhang, F. Cui, and K. Li, "Compact Ho robust rebalance loop controller design for a micromachined electrostatically suspended gyroscope," ISA Transactions, vol. 49, no. 2, pp. 222-228, 2010.

[16] J. Liu, Advanced PID Control and Its Matlab Simulation, Electronic Industry Press, Beijing, China, 2004.

[17] P. Ioannou and J. Sun, Robust Adaptive Control, Prentice-Hall, Upper Saddle River, NJ, USA, 1996. 


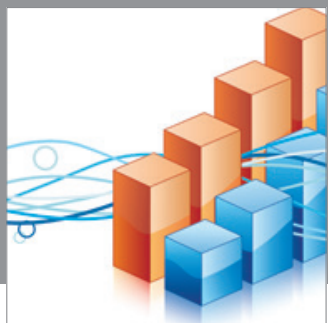

Advances in

Operations Research

mansans

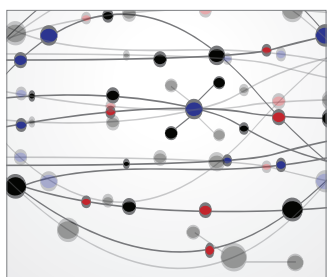

The Scientific World Journal
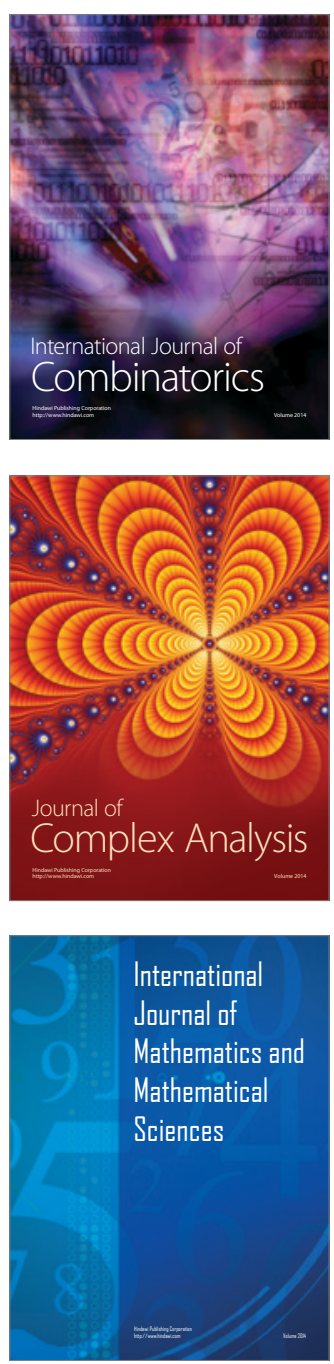
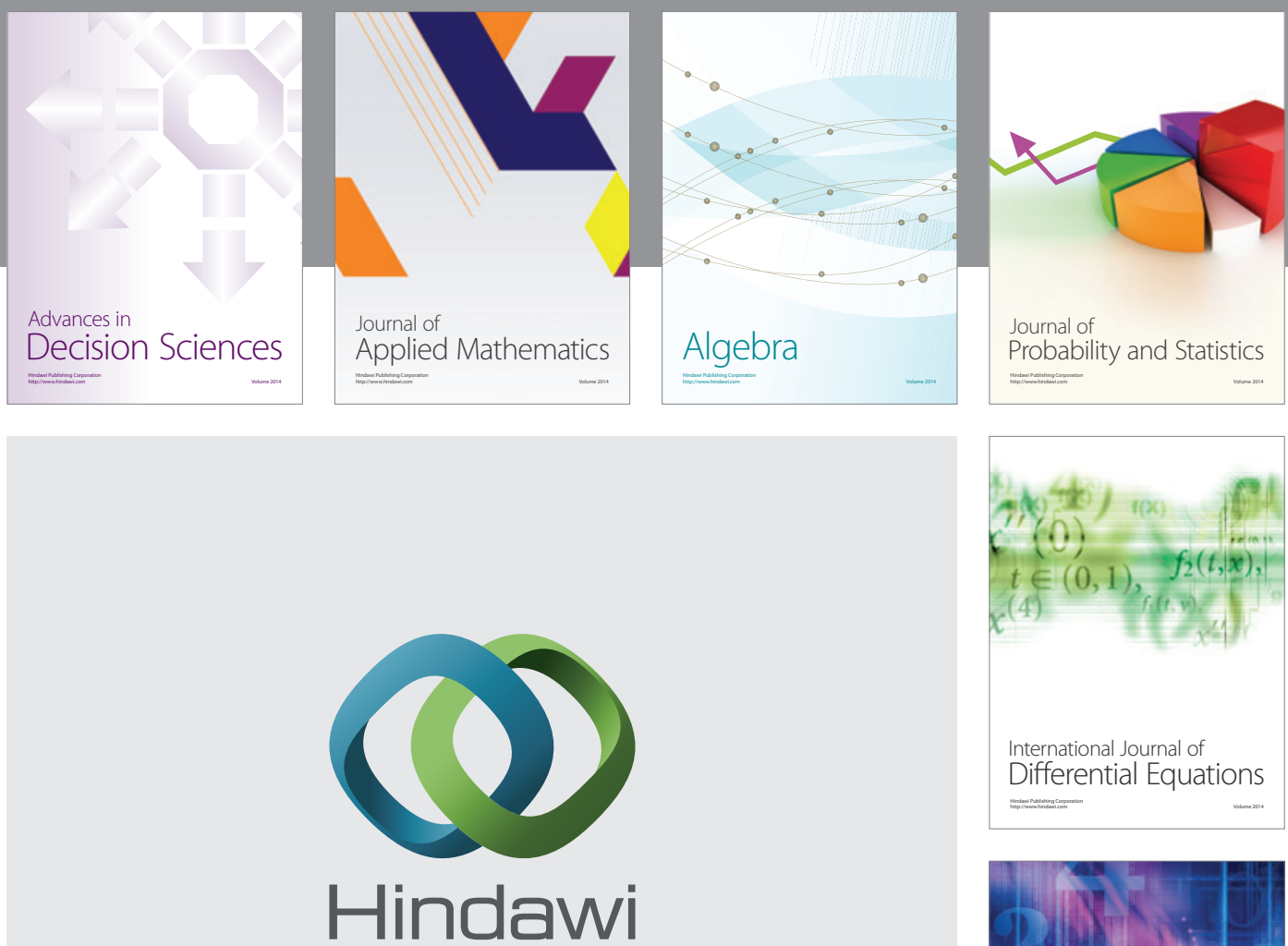

Submit your manuscripts at http://www.hindawi.com
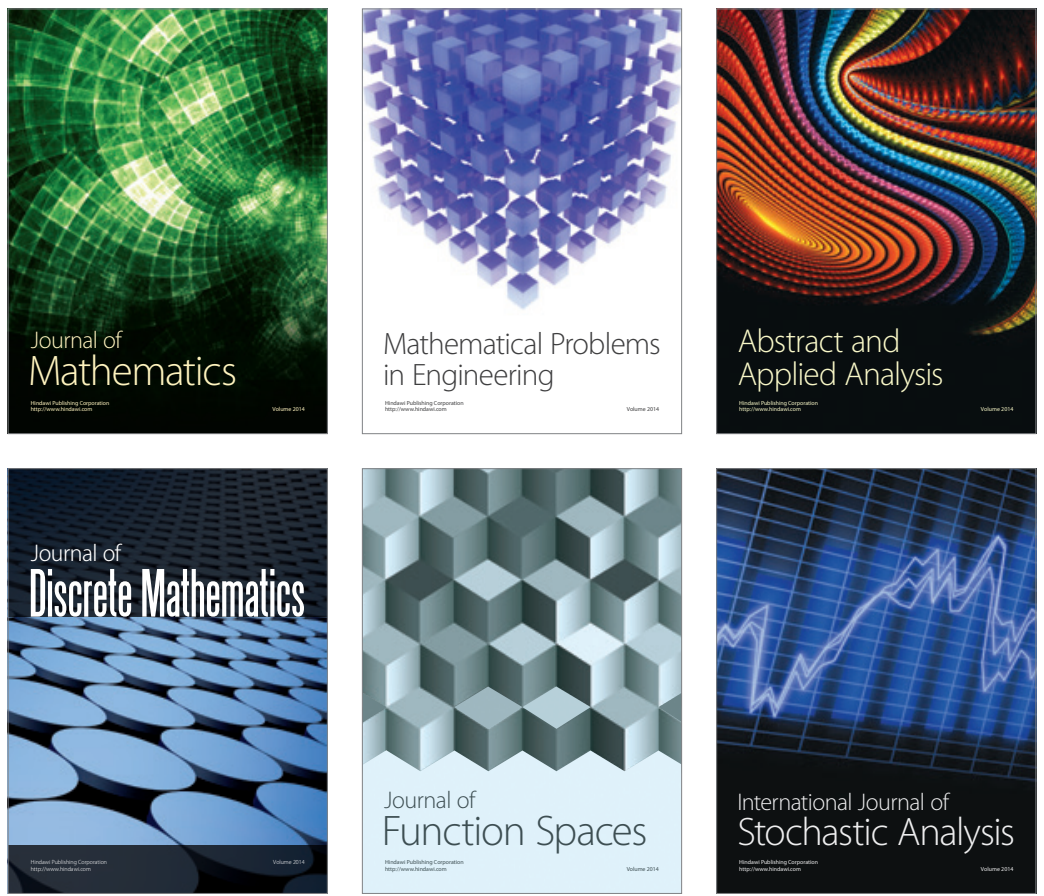

Journal of

Function Spaces

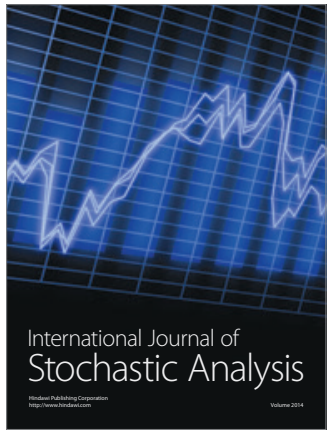

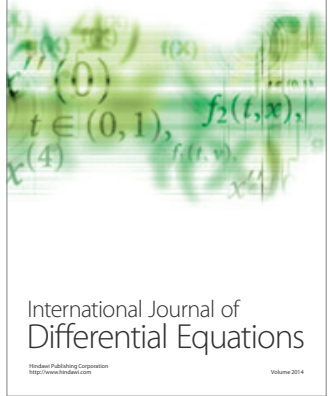
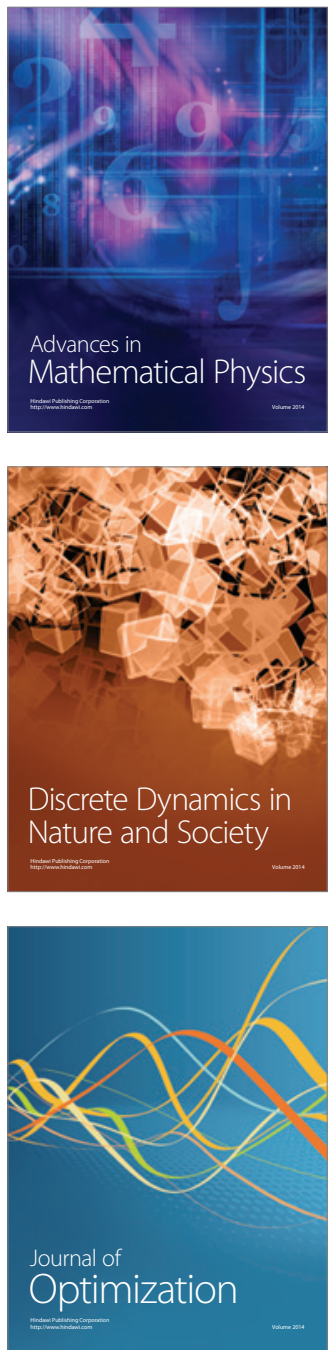